\title{
Diabetes Mellitus: A New Look at Diagnostic Criteria
}

\section{The Editor, Diabetologia}

Dr. Peter Watkins in his comments on our suggestions for revised blood glucose criteria for diabetes mellitus (Diabetologia 17, 127-128, 1979) agrees that the diagnostic levels should be raised, but proposes that those people not attaining them should be regarded (with the exception of the pregnant) as normal. If only the world were black and white, diabetic and non diabetic, hypertensive and nonhypertensive how much simpler things would be. But it is not; with blood glucose as with many other biological variables in human populations, we have to deal with the phenomenon of 'continuous variation', a smooth transition of the frequency distribution from the majority who are clearly normal through those with steadily diminishing frequency and increasing probalitiy of disease to the small number who are quite clearly abnormal. It is this probabalistic approach to diagnosis rather than the iron-hard, somewhat old fashioned, plus or minus formulation, that best fits the data that are available.

We agree wholeheartedly that the oral glucose tolerance test is performed much too often, frequently in a mindless way, when a single unequivocally raised blood glucose value accompanied by symptoms has already established the diagnosis of diabetes. Nevertheless these tests are done and we have to make decisions and interpretations (often for others) on the results. We cannot accept Dr. Watkin's robust view that the diagnosis of diabetes does little damage. Perhaps the only reasonably certain thing about a diagnosis is that it will damage its recipient; the justification can only be that the value of treatment compensates the damage done. For the subject with "impaired glucose tolerance" (I. G. T.) (not diabetes) clearly effective treatment remains to be demonstrated. None the less, I. G. T. cannot be ignored. It is more likely, on average, to 'worsen to diabetes' than normal glucose tolerance and it is associated with a greater risk of cardiovascular disease and death. We may not at present be able to prevent the metabolic deterioration, but we can be ready for it; and there are reversible factors for arterial disease present in excess in this group which can be tackled.
The view that insurance companies would not like new criteria because they differ from their ancient practices is not one that we feel should be given too much weight in determining what is right. We must hope that they will themselves respond to the logic of the case and modify their policies accordingly.

Impaired glucose tolerance in pregnancy is clearly a serious matter. There is, we suspect, at present much confusion and complexity in the rather variable advice and recommendations given to obstetricians. We feel that, far from further obscuring the issue, the new criteria offer a great simplification - in pregnancy, treat IGT as diabetes.

Regarding the glucose load, Dr. Watkins claims that "any agreement to change the load might not be universally adopted". The U.S., Australia, some European countries and others elsewhere are actually using it or are well on the way to adopting it. One thing is certain and that is that if no one makes a move the present chaos will continue. It is not just for the advantage of epidemiologists to standardise the load. At least part of the variation in the risk of being diagnosed diabetic from country to country (or from street to street in the same country) is attributable to variation in the loading dose of glucose.

Dr. Watkins questions the ability of his colleagues to grasp the new criteria. Most of their difficulties must surely be attributable to the confusion of the many sets of criteria currently purveyed. A single new set, based upon a reasoned review of the relevant evidence, nationally and in due course internationally accepted, is surely the only antidote to the prevailing confusion.

Yours sincerely,
H. Keen ${ }^{1}$
R. J. Jarrett ${ }^{1}$
K. G. M. M. Alberti ${ }^{2}$

\footnotetext{
1 Unit for Metabolic Medicine

Department of Medicine

Guy's Hospital Medical School

London SEI

England

${ }^{2}$ Department of Clinical Biochemistry

Royal Victoria Infirmary

Newcastle upon Tyne NE1 4LP

England
} 